\title{
ИССЛЕДОВАНИЕ ПРОИНСУЛИНА В ДИАГНОСТИКЕ НЕДИАБЕТИЧЕСКИХ ГИПОГЛИКЕМИЙ
}

\author{
Юкина М.Ю., Нуралиева Н.Ф., Трошина Е.А., Платонова Н.М.
}

ФГБУ «Национальный медицинский исследовательский чентр эндокринологии» Минздрава России

Согласно клиническим рекомендациям Endocrine Society от 2009г, при завершении пробы с голоданием для диагностики недиабетической гипогликемии (НДГ) и ее вариантов (гипер- или гипоинсулинемический) необходимо исследование уровня инсулина (И), С-пептида (С-п) и проинсулина (П). В то же время, ряд экспертов рекомендует выполнять исследование П селективно, только при дискордантных уровнях основных гормонов (И и С-п). Также, расходятся мнения специалистов относительно диагностического порога П - 5 или 22 пмоль/л.

ЦЕЛЬ: 1) определить когорту пациентов, нуждающихся в исследовании уровня П при завершении пробы с голоданием; 2) определить диагностический порог П, позволяющий дифференцировать гипери гипоинсулинемические варианты НДГ.

МАТЕРИАЛЫ И МЕТОДЫ: Включено 388 пациентов 18-80 лет с подозрением на НДГ (группа 1). Всем участникам выполнена проба с голоданием для верификации наличия НДГ и определения ее варианта. При завершении пробы выполнялось исследование глюкозы, И и С-п венозной крови. При гиперинсулинемическом варианте НДГ проводился топический поиск инсулин-продуцирующей опухоли. В группе 1 уровень П исследован при завершении пробы 98 пациентам с подтвержденной НДГ (группа 2).

РЕзультАТЫ: 1) в группе 1 по результатам пробы с голоданием лишь у 17 пациентов $(4,4 \%)$ вариант НДГ определить не удалось из-за дискордантных уровней И и С-п; 2) в группе 2 уровень П у пациентов с гиперинсулинемическим вариантом НДГ (n=82) составил 17,6 [5,3; 56,9], $(0,5,557,4)$ пмоль/л, из них с инсулиномой $(n=73)-29,2$ [7,6; 63,1], $(2,2,557,4)$ пмоль/л, а у пациентов с другими причинами НДГ $(\mathrm{n}=9)-1,5[1,4 ; 2,2](0,5,4,8)$ пмоль/л; при гипоинсулинемическом варианте НДГ $(\mathrm{n}=16)-1,4[0,8 ; 2,0]$, $(0,5,3,7)$ пмоль/л. По данным ROC-анализа, диагностический уровень, позволяющий дифференцировать гиперинсулинемический вариант НДГ от гипоинсулинемического, составил 2,84 пмоль/л, Se 87,3\%, 95\% ДИ [78,0\%; 93,1\%], Sp 93,8\%, 95\% ДИ [69,4\%, 100\%] (отличное качество модели: AUC 94,1\%, 95\% ДИ [89,7\%; 98,6\%]). Диагностический уровень, позволяющий дифференцировать инсулиному от гиперинсулинемической НДГ другого генеза, составляет 5,02 пмоль/л, Se 87,1\%, 95\% ДИ [77,0\%; 93,2\%], Sp 100,0\%, 95\% ДИ [65,0\%, 100\%] (отличное качество модели: AUC 97,6\%, 95\% ДИ [94,3\%; 100,0\%]).

\section{выводы:}

1. анализ крови на П на фоне гипогликемии целесообразно выполнять избирательно пациентам с неоднозначными результатами исследования И и С-п, а также пациентам с гиперинсулинемическим вариантом НДГ, но с отрицательными визуализирующими методами 1 ряда (КТ с контрастированием или МРТ и УЗИ органов брюшной полости). Селективное исследование П позволит уменьшить стоимость обследования пациента;

2. применение диагностического порога П 22 пмоль/л приводит к снижению чувствительности метода и не может быть рекомендовано для клинической практики. Для дифференциальной диагностики инсулиномы с другими вариантами НДГ (гиперинсулинемического иного генеза и гипоинсулинемического) диагностический порог составляет 5 пмоль/л, а для неинсулиномного гиперинсулинемического варианта НДГ - 2,8 пмоль/л. 